\title{
23. Gentechnische Anwendungen im Spiegel der nachhaltigen Entwicklung
}

\subsection{Einleitung}

Das Verhältnis von Gentechnik und Nachhaltigkeit ist von vielen Unsicherheiten und Unklarheiten geprägt. Die etablierte Strukturierung in ökologische, ökonomische und soziale Nachhaltigkeit bietet sich an, um die wesentlichen Auswirkungen unterschiedlicher gentechnischer Anwendungen auf eine nachhaltige Entwicklung zu überprüfen und zu kategorisieren (Gottwald, 2011; Niggli, 2012; EASAC, 2013; Rösch et al., 2020). Vor allem geht es darum, die Ambivalenz gentechnischer Innovationen in Bezug auf ihre Nachhaltigkeitsbilanz aufzuzeigen. Aufgrund der Vielzahl an Gestaltungsmöglichkeiten hängt es weitgehend von der jeweiligen Anwendung der Gentechnik als Querschnittstechnologie ab, ob und in welchem Maße nachhaltige Ziele erreicht werden können (Clément/Ajena, 2021).

In den folgenden Abschnitten steht die Kompatibilität gentechnischer Anwendungen in Bezug auf die ökologische, ökonomische und soziokulturelle Komponente der Nachhaltigkeit im Vordergrund. Dabei geht es um eine Einordnung der Chancen und Risiken der Gentechnik im Zusammenhang mit den Zielen der Nachhaltigkeit, wie sie etwa in den von der UN verabschiedeten Sustainable Development Goals (SDGs) zum Ausdruck kommen (UN, 2019; One Planet Network, 2020: 67/73).

\subsection{Klassifizierung gentechnischer Anwendungen}

Bei der Gentechnologie handelt es sich um eine sogenannte Querschnittstechnologie. Die Anwendungsbereiche der Gentechnologie lassen sich grob in vier große Felder unterteilen (Steinhoff, 2005; Matyushenko et al., 2016):

Ein großes Feld ist die medizinische und pharmazeutische Anwendung, die oft als „rote“ Gentechnik bezeichnet wird (zum wissenschaftlichen Sachstand siehe Fehse, Kap. 6). In der Medizin liegen die Anwendungsfelder vor allem in der Therapie und in 
der Diagnose, in der Pharmazie wird die Gentechnik in erster Linie für die Entwicklung und auch die Herstellung von Arzneimitteln verwendet. Bereits heute wird mit gentechnisch erzeugten Alpha-Interferonen die Leukämie und mit Beta-Interferonen die multiple Sklerose bekämpft und mit gentechnisch hergestellten Seren ein verbesserter Schutz gegen Hepatitis geboten. Diese und weitere Anwendungen sind zwar auch mit Risiken verbunden, der Nutzen der Anwendungen überwiegt aber bei weitem die möglichen Gesundheitsrisiken. Dass Medikamente Nebenwirkungen haben können, ist kein Spezifikum gentechnisch hergestellter Pharmazeutika. Inwieweit von der Produktion gentechnisch erzeugter Pharmazeutika spezifische Risiken (z. B. Entweichung aus dem Labor) ausgehen, ist weiterhin umstritten; das Ausmaß dieser Risiken kann aber als relativ unbedeutend und regional begrenzt angesehen werden.

Wesentlich kritischer ist dagegen der direkte gentechnische Eingriff am Menschen $z u$ werten, etwa in der Keimbahntherapie. Vor allem in der Diagnose von Krankheiten im pränatalen Stadium sowie in der Reproduktionsmedizin werden zentrale ethische Fragen aufgeworfen (Luger et al., 2017). Auf diese Anwendungen soll aber hier nicht weiter eingegangen werden. Sie sind in anderen Stellungnahmen, auch in diesem Band (siehe Fehse et al., Kap. 9), bereits vielfach kommentiert worden.

Das zweite große Betätigungsfeld der Gentechnologie liegt in der Landwirtschaft, das meist als „Grüne“ Gentechnik bezeichnet wird (Müller-Röber et al., 2013; zum aktuellen Forschungsstand siehe Clemens, Kap. 7). In der agrokulturellen Anwendung geht es beispielsweise um gentechnisch veränderte Rohstoffe zur Nahrungsmittelproduktion, Resistenzzüchtungen, Intensivierungszüchtungen, Produktivitätssteigerungen, Qualitätsveränderungen und Anreicherungen mit ernährungsphysiologisch erwünschten Zusatzstoffen (etwa Vitaminen). Auch Anwendungen in der Tierzucht werden meist der grünen Gentechnik zugeordnet. Auf diese Anwendungen soll in den folgenden Abschnitten vorrangig eingegangen werden.

Zum Dritten findet man gentechnische Verfahren in speziellen Produktionsprozessen, z. B. bei der Enzymproduktion für Waschmittel. Auch für die Herstellung von Zwischenprodukten für die chemische Synthese können gentechnische Verfahren eingesetzt werden. Dieser Anwendungsbereich ist wenig spektakulär, da die Verfahren selten mit Endprodukten für Konsumentinnen und Konsumenten verbunden sind. Dieser Anwendungsbereich wird häufig als ,weiße“ Gentechnik bezeichnet (Luger et al., 2017: 21).

Zum Schluss findet man gentechnische Verfahren auch im Bereich der Umwelttechnik und Schadstoffbeseitigung. Hierzu zählt unter anderem die sogenannte „Bioremediation“. Darunter versteht man den Einsatz von Mikroorganismen zur Entsorgung kontaminierter Böden und Gewässer. Dieser Anwendungsbereich wird meist als ",graue“ Gentechnik bezeichnet (McCormick/Kautto, 2013). 
Darüber hinaus findet sich gelegentlich auch die Bezeichnung: „blaue“ Gentechnik. Sie umfasst gentechnische Anwendungen im Meer (Luger et al., 2017: 21).

Für die Bewertung im Hinblick auf Nachhaltigkeit ist vor allem die Grüne Gentechnik von Bedeutung. Zum einen hat sie mehr als die anderen Anwendungsfelder Auswirkungen auf alle drei Komponenten der Nachhaltigkeit, zum anderen wird von Gruppen, die der Gentechnik kritisch oder skeptisch gegenüberstehen, gerade die Nachhaltigkeit der grünen Gentechnik in Frage gestellt. Daher wird im Folgenden die Grüne Gentechnik auf ihre Wirkungen im Hinblick auf Nachhaltigkeit unter die Lupe genommen.

\subsection{Die Grüne Gentechnik}

Die Grüne Gentechnik umfasst alle Anwendungen der Gentechnik für die Felder Landwirtschaft, Ernährung und Produktion von Biomasse (für Energie und Rohstoffe). Eine wichtige Motivation für gentechnische Modifikationen ist die Tatsache, dass die bei jeder Züchtung unvermeidbaren Nebenfolgen im Sinne von nicht erwünschten, aber durch weitere Kreuzung unvermeidbaren Merkmalen (etwa geringe Halmfestigkeit) durch gezielte gentechnische Eingriffe vermieden werden können. Gentechnische Veränderungen sind gezielter und treffgenauer als konventionelle Züchtungserfolge (Akademien der Wissenschaften Schweiz, 2013). Das gilt vor allem für Pflanzen, die nach der neuen CRISPR/Cas-Methode entwickelt wurden (Gao, 2018; Metha et al., 2020). Mithilfe der Gentechnik können Nutzpflanzen (und auch Nutztiere) stärker als bisher auf die gewünschte Nutzwirkung hin optimiert und gleichzeitig die bei Züchtungen unvermeidbaren Nebenfolgen weitgehend vermieden werden (Wolter et al., 2019).

Mittels Gentechnik können auch erwünschte Eigenschaften wie Pestizidresistenz oder Schädlingsresistenz in die Pflanze „eingebaut“ werden (Morawicki/Diaz Gonzalez, 2018). Bei den gentechnisch veränderten Pflanzen der ersten und zweiten Generation (1970 bis 2010) wurden vor allem solche Eigenschaften gefördert bzw. initiiert, die ertragssteigernd wirkten oder in Anbau, Ernte, Transport und Lagerung wirtschaftliche Vorteile versprachen. In der zweiten und dritten Generation (ab 2010) werden auch spezifische Inhaltsstoffe entweder vermieden, wenn sie unerwünschte Folgen mit sich bringen, oder zugeführt, wenn damit ernährungsphysiologisch erwünschte Eigenschaften wie erhöhter Nährstoffgehalt oder Anreicherung mit bestimmten Spurenstoffen erzeugt werden kann. Ein prominentes Beispiel ist die Vitamin-A-Anreicherung beim sog. „Golden Rice“ (Tang et al., 2009; Dubock, 2019; siehe auch van den Daele/ Broer, Kap. 21).

Diese potenziellen Vorteile werden von Kritikerinnen und Kritikern wie Befürworterinnen und Befürwortern anerkannt, allerdings unterschiedlich gewichtet. Während 
die Befürworterinnen und Befürworter diese Anwendungen als einen wichtigen Fortschritt für die Sicherung der weltweiten Ernährung im Umfeld großer Unsicherheiten (Klimawandel, Wasserverknappung, etc.) beurteilen (so auch van den Daele/Broer, Kap. 21), sind die Kritikerinnen und Kritiker überwiegend der Ansicht, dass die Anwendungen vor allem Groß-Produzenten (Agrobusiness) und multinationale Unternehmen begünstigen, aber weder für kleinere Familienbetriebe noch für Verbraucherinnen und Verbraucher Vorteile bringen. Auch die Welternährungslage ließe sich mit Gentechnik nicht nennenswert verbessern (Gottschlich et al., 2017; CSS et al., 2019).

Bei der Frage nach den Risiken gentechnischer Anwendungen sind die Meinungen der Kritikerinnen und Kritiker sowie der Befürworterinnen und Befürworter der grünen Gentechnik stärker polarisiert als bei der Einschätzung der Vorteile. Im Vordergrund der Debatte stehen dabei potenzielle langfristige Gesundheitsschäden und die Möglichkeit eines horizontalen Gentransfers (Morawicki/Diaz Gonzalez, 2018; Islam et al., 2020). Im ersten Falle liegt das Risiko in den möglichen Nebenwirkungen der gentechnisch modifizierten Nahrungsmittel auf die menschliche Gesundheit, im zweiten Falle in der Reduktion der Biodiversität (Busch et al., 2002: 66). Das Risiko beim Gentransfer liegt vor allem darin, dass Eigenschaften von Nutzpflanzen, die gentechnisch verändert wurden, durch Auskreuzen auf Wildpflanzen übertragen werden. Unter Umständen können sich die dann entstehenden neuen Wildpflanzen besser ausbreiten und dabei andere Pflanzen verdrängen. Dies könnte negative Folgen für die Biodiversität und die Verwundbarkeit von fragilen Ökosystemen haben (Carpenter, 2011).

Der Einsatz der Gentechnik ist also mit Chancen und Risiken verbunden. Welche Chancen und welche Risiken aber überwiegen, lässt sich pauschal nicht feststellen, sondern erfordert eine Abwägung im Einzelfall. Herbizidresistente Pflanzen sind sicherlich anders zu bewerten als gentechnisch veränderte Pflanzen, die eigene Abwehrstoffe gegen einen Insektenbefall entwickeln oder die ernährungsphysiologisch wichtige Nährstoffe anreichern. Zudem ist bei jeder Abwägung eine Gewichtung der eingesetzten Kriterien notwendig, die auf der Basis von Wertprioritäten festgelegt werden müssen. Welche Werte aber Priorität gegenüber anderen haben sollen, ist keine Frage der Wissenschaft, sondern der gesellschaftlichen Aushandlung. Dazu kommt noch, dass sich viele Risiken in ihrer Qualität nicht von den Risiken der herkömmlichen Pflanzenzüchtung unterscheiden. Gentechnische Verfahren haben aber das Potenzial, die Entwicklung solcher Risiken zu beschleunigen oder ihr Ausmaß zu verstärken. Gleichzeitig sind mit dem Einsatz gentechnisch veränderter Pflanzen viele Vorteile verbunden, die zu dem Ziel einer ausreichenden und qualitativ hochwertigen Ernährung der Bevölkerung beitragen können (Braun et al., 2013: 18). Dabei ist jedoch immer zu berücksichtigen, dass die Ernährungskrisen der Menschheit weniger das Resultat mangelnder Nah- 
rungsmittel sind als vielmehr Folge verfehlter Landwirtschafts- und Verteilungspolitik oder sich zwangsweise als Konsequenz der mangelnden Kaufkraft, also der weltweiten Armut ergeben (Scholz et al., 2021). Daran werden auch verbesserte gentechnische Verfahren und Produkte wenig ändern.

\subsection{Die Nachhaltigkeitsbilanz der Grünen Gentechnik}

Das übergeordnete Ziel der nachhaltigen Entwicklung innerhalb der Landwirtschaft bietet sich als Leitbild für eine kohärente und am Gemeinwohl orientierte Bewertung gentechnischer Anwendungen an. Wichtig ist dabei, dass alle drei Komponenten der Nachhaltigkeit, die ökologische, wirtschaftliche und soziale Komponente, einbezogen und in ihren jeweiligen Wechselwirkungen analysiert werden (Rösch et al., 2020). Im Folgenden werden diese drei Komponenten näher ausgeführt und die damit verbundenen Implikationen für die Bewertung gentechnischer Anwendungen nach den Kriterien der Nachhaltigkeit abgeleitet.

\subsubsection{Die ökologische Komponente der Nachhaltigkeit}

Im Rahmen der ökologischen Nachhaltigkeit stehen drei Ziele im Fokus: Dekarbonisierung, Dematerialisierung und Renaturalisierung zum Erhalt der natürlichen Biodiversität (Altieri, 2018; Renn et al., 2021). Das Ziel der Dekarbonisierung umfasst die Reduktion von klimaschädlichen Gasen, vor allem $\mathrm{CO}_{2}$, aber auch anderen Klimagasen wie Methan oder Lachgas. Diese Treibhausgase entstammen überwiegend aus Anlagen zur Energieversorgung, aber auch in einem erheblichen Ausmaß aus der landwirtschaftlichen Praxis (Entwaldung, Entwässerung von Feuchtgebieten, Lachgas-Emissionen, Methan-Emissionen). Bei der Dematerialisierung wird das Ziel verfolgt, wirtschaftliche Produkte und Dienstleistungen mit einem Minimum an Materialeinsatz, Abfall und Emissionen zu erzeugen und dort, wo es nicht vermeidbar ist, auf umweltverträgliche Materialien oder Prozesse auszuweichen. Schließlich umfasst das Ziel der Renaturalisierung den Erhalt der Biodiversität und den Fortbestand naturnaher Ökosysteme, aber auch den Erhalt der Vielfalt bei den Kulturpflanzen und Nutztieren. Was die Gentechnik anbetrifft, lassen sich viele Chancen, aber auch Risiken im Hinblick auf diese drei ökologischen Ziele aufführen. Zunächst die Chancen:

a. Gentechnische Anwendungen können dazu beitragen, die Emissionen an $\mathrm{CO}_{2}$, Lachgas und Methan zu reduzieren (James, 2010; Lotz et al., 2020). Pflanzen können gentechnisch so verändert werden, dass sie beispielweise mit weniger Düngemitteln (und einem insgesamt geringeren Einsatz von Herbiziden oder Pestiziden) auskom- 
men. Allerdings ist der mögliche Beitrag der Gentechnik zur Dekarbonisierung begrenzt; viel entscheidender sind hier die Art der Landnutzung, der Viehbestand und die eingesetzten Anbaumethoden.

b. Gentechnische Anwendungen können zur Effizienzverbesserung bei der Erzeugung von Biokraftstoffen beitragen. Sie dienen dabei der schnelleren Umsetzung der Energiewende in Richtung einer nachhaltigen Energieversorgung. So werden inzwischen mithilfe der Genschere CRISPR/Cas9- mikrobielle Zellen zur verbesserten Produktion von Biokraftstoffen entwickelt (Nymark et al., 2016).

c. Nutzpflanzen, vor allem Getreidesorten, können gentechnisch so modifiziert werden, dass sie die beiden Funktionen der Erzeugung von hochwertigen Nahrungsmitteln und der Produktion von Biomasse zur Vergärung als Komponente einer nachhaltigen Energieversorgung optimal verbinden (Busch et al., 2002: 71). Dadurch kann der Konflikt „Nahrung versus Energie“ entschärft werden.

d. Prinzipiell können gentechnisch veränderte Pflanzen höhere Erträge erbringen, was eine Ausweitung landwirtschaftlicher Flächen auf bislang naturnahe Räume begrenzen kann (Bailey-Serres et al., 2019; Islam et al., 2020: 1679).

Der Einsatz von Gentechnik in der Landwirtschaft erzeugt aber auch Risiken im Hinblick auf die ökologische Nachhaltigkeit:

a. In der Literatur ist es umstritten, ob der Einsatz von Gentechnik zu einer NettoReduktion von Düngemitteln und von Pestiziden bzw. Herbiziden beiträgt. Dabei ist inzwischen belegt, dass z. B. Ackerflächen mit herbizidresistenten Pflanzen weniger Herbizide benötigen, um den gewünschten Effekt zu erzielen. ${ }^{1}$ Aber die weltweite Ausweitung herbizidresistenter Kulturen kann den Einsatz von Herbiziden absolut

1 „Eine kürzlich durchgeführte Meta-Analyse von 147 veröffentlichten Studien zu gentechnisch veränderten Pflanzen aus den Jahren 1995 bis 2014 kam zu dem Schluss, dass solche Pflanzen in den letzten 20 Jahren vielfältige und greifbare Vorteile gebracht haben. Laut dieser Studie hat die Einführung der GVO-Technologie im Durchschnitt den Einsatz von chemischen Pestiziden um 37 Prozent reduziert, die Ernteerträge um 22 Prozent erhöht und die Gewinne der Landwirte um 68 Prozent gesteigert" (Klümper et al., 2014, zitiert nach Morawicki/Diaz Gonzalez, 2018; Übersetzung durch den Verfasser). „Die Einführung von GV-insektenresistenten und herbizidtoleranten Pflanzen hat das Sprühen von Pestiziden um 581,4 Millionen kg (8,2 Prozent Reduktion) reduziert, und die mit dem Herbizid- und Insektizideinsatz bei diesen Kulturen verbundenen Umweltauswirkungen sind gemessen durch den EIQ-Indikator seit 1996 um 18,5 Prozent gesunken“ (Brooks/Barfoot, 2016, zitiert nach Morawicki/Diaz Gonzalez, 2018; Übersetzung durch den Verfasser). 
gesehen begünstigen und andere (biologisch verträglichere) Formen der Unkrautkontrolle verdrängen. ${ }^{2}$

b. Die zu beobachtende Tendenz, universell einsatzfähige gentechnisch modifizierte Pflanzen zu erzeugen, kann zu an die jeweiligen lokalen Bedingungen wenig angepassten Anbaustrukturen führen, die wiederum negative Auswirkungen auf die Biodiversität mit sich bringen können (Busch et al., 2002: 66 ff.). Dieses Risiko ist weniger ein Argument gegen Gentechnik per se (gentechnische Modifikationen können ja auch der Angepasstheit an lokale Gegebenheiten dienen) als eine Kritik an der zu beobachtenden Tendenz der großen Anbieter von gentechnisch veränderten Pflanzen, mit einem begrenzten Spektrum an Produkten den Weltmarkt abzudecken.

c. Wenn wenige Getreidearten durch gentechnische Veränderung so widerstandsfähig und ertragreich werden, dass sie weltweit Verbreitung finden und die Weltgetreideversorgung von ihnen abhängen würde, könnten neue, bisher noch unbekannte Schädlinge oder Virenformen diese Versorgung global gefährden (CSS et al., 2019). Dieses Szenario ist zwar auch bei konventioneller Züchtung gegeben, aber mit dem Einsatz der Gentechnik kann dieser Trend zu einigen dominanten Nutzpflanzen noch wesentlich beschleunigt werden und die Biodiversität bei den Kulturpflanzen erheblich reduzieren (Busch et al., 2002: 66).

d. Ein möglicher Gentransfer von Kultur- auf Wildpflanzen könnte zu einer Verringerung der Biodiversität und einer schwerwiegenden Veränderung der Ökosysteme führen (Braun et al., 2013: 36 ff.). Dieses Risiko wird von den meisten Expertinnen und Experten für sehr gering eingestuft, weil Kulturpflanzen selten Eigenschaften aufweisen, die sie in der Wildnis überleben lassen, aber in einzelnen Fällen ist ein solcher Transfer auch nicht ausgeschlossen (Morawicki/Diaz Gonzalez, 2018). In Extremfällen könnte dies zu einer exponenziell wachsenden Verbreitung besonders angepasster Arten führen (Islam et al., 2020: 1680).

Fazit: Die Anwendung gentechnischer Verfahren hat nur einen geringen Effekt auf die beiden Unterziele der ökologischen Nachhaltigkeit: Dekarbonisierung und Dematerialisierung. In beiden Fällen gibt es geringe Vorteile aber auch Risiken, je nachdem

2 „Allerdings hat durch den zunehmenden Anbau von Glyphosat-toleranten GV-Nutzpflanzen die großflächige Anwendung dieses Pflanzenschutzmittels auf globaler Ebene stark zugenommen. Statt einer Kombination verschiedener Wirkstoffe wird häufig nur noch Glyphosat verwendet. Dies erhöht einerseits das Risiko von Resistenzentwicklungen in Unkräutern und führt andererseits zu einer höheren Belastung der Umwelt mit einem einzelnen Wirkstoff“" (Braun et al., 2013: 39; siehe auch Coyette et al., 2002). 
welche Anwendungen man betrachtet (Niggli, 2012; Godfray, 2015). Bei der Frage der Renaturalisierung und der Biodiversität ergeben sich aber größere Chancen und Risiken. Wenn gentechnisch veränderte Pflanzen gezielt auch auf nährstoffärmeren Böden Erträge sicherstellen, den jeweiligen ökologischen Bedingungen am Einsatzort angepasst sind und kaum oder keine Chancen für eine Auswilderung bestehen, können sie zum dritten Ziel der ökologischen Nachhaltigkeit substanziell beitragen (EASAC, 2013). Wenn diese Bedingungen nicht erfüllt sind, ist der Beitrag entweder vernachlässigbar oder sogar negativ. Das gilt vor allem für gentechnische Pflanzen, bei denen das Risiko eines horizontalen Gentransfers besteht (Busch et al., 2002: 63).

\subsubsection{Die ökonomische Komponente der Nachhaltigkeit}

Im Rahmen der ökonomischen Komponente der Nachhaltigkeit stehen ebenfalls drei Ziele im Vordergrund: die Versorgung der Menschheit mit basalen Gütern und Dienstleistungen (hier vor allem der Ernährung), die Förderung der Kreislaufwirtschaft und die Sicherstellung langfristiger und sozial abgesicherter Beschäftigungs- und Eigentumsverhältnisse (Gliessman, 2015; Renn et al., 2021). Das Thema Grundversorgung und Versorgungssicherheit ist dabei primäres Ziel des Wirtschaftens überhaupt. Die Leitidee der Kreislaufwirtschaft ist eng mit den oben behandelten ökologischen Zielen verknüpft. Nach dem Prinzip „von der Wiege bis zur Bahre“ sind alle Stationen der Wertschöpfungskette zu beachten (Zink/Geyer, 2017). Das dritte Ziel der langfristig gesicherten Arbeitsverhältnisse ist im Bereich der Landwirtschaft vor allem auf die Existenzfähigkeit bäuerlicher Betriebe ausgerichtet. Ähnlich wie bei der ökologischen Komponente lassen sich hier wieder Chancen und Risiken der Gentechnik aufzeigen. Zunächst die Chancen:

a. Die Befürworterinnen und Befürworter der Gentechnik sehen in dieser Querschnittstechnologie vielfache Möglichkeiten, eine wachsende Weltbevölkerung ohne Ausweitung der landwirtschaftlich genutzten Flächen ausreichend zu ernähren (Breeman et al., 2017; Zaidi et al., 2019; siehe van den Daele/Broer, Kap. 21). Dabei können gentechnisch veränderte Pflanzen zum einen mehr Erträge liefern, an neue klimatische Anforderungen angepasst und gegenüber Konkurrenzpflanzen und Fressfeinden geschützt werden. Dieses Potenzial wird von vielen Kritikerinnen und Kritikern der Gentechnik bestritten oder zumindest als wenig effektive Form der Sicherung der Ernährung für alle herausgestellt (Vogt/Hagemann, 2010).

b. Die Ziele der Kreislaufwirtschaft werden durch den Einsatz der Gentechnik weder positiv noch negativ berührt. Allenfalls könnten durch den verminderten Einsatz von Agro-Chemikalien weniger Abfälle, die nicht biologisch abbaubar sind, anfallen. 
Dies setzt aber voraus, dass auch deutlich weniger Pestizide und Herbizide bei gentechnisch veränderten Pflanzen verwendet werden (Lotz et al., 2020).

c. Da durch den Einsatz von gentechnisch veränderten Pflanzen die Erträge gesteigert und damit auch die Einkommen der in der Landwirtschaft Tätigen erhöht werden können, kann die Gentechnik, zu einer verbesserten und dauerhaften Existenzsicherung der landwirtschaftlichen Betriebe beitragen (Müller-Röber et al., 2013; Kellermann, 2020a). Wie später bei den Risiken ausgeführt, wird dieser Einkommenseffekt aber von vielen Kritikerinnen und Kritikern der Gentechnik nur bei den Großbetrieben gesehen.

Auch bei der ökonomischen Nachhaltigkeit lassen sich Risiken der Gentechnik aufzeigen:

a. Der Anspruch, dass Gentechnik zur Sicherung der Ernährung der Weltbevölkerung beitrage, geht an den eigentlichen Ursachen der mangelnden Versorgung vor allem ärmerer Bevölkerungsteile vorbei. Wesentliche Treiber sind hier die hohe Inanspruchnahme landwirtschaftlicher Flächen zur Fleischerzeugung (Viehhaltung), die ungleiche Verteilung von Ressourcen zwischen den Ländern und innerhalb eines Landes, die ungleichen Eigentumsverhältnisse und die Handelspolitik der mächtigen Länder (Vogt/Hagemann, 2009; Montenegrode, 2020). Durch den hohen Industrialisierungs- und Konzentrationsgrad, der mit Gentechnik verbunden ist, könnten diese Ungleichgewichte noch verstärkt und die Ernährung vor allem der ärmeren Schichten der Bevölkerung zunehmend gefährdet werden (IPES-Food, 2016).

b. Wie schon oben ausgeführt, sind die Bemühungen zur Kreislaufwirtschaft in der Landwirtschaft nur marginal mit der Frage der Gentechnik verbunden. Man könnte allenfalls als mögliches Risiko aufzählen, dass durch die Abhängigkeit der landwirtschaftlichen Betriebe von großen agro-industriellen Konzernen die bestehenden Strukturen der konventionellen Landwirtschaft gestärkt werden, die einer konsequenten Umsetzung des Kreislaufgedankens entgegenstehen (Busch et al., 2002: 71).

c. Der Einsatz der Gentechnik verspricht betriebswirtschaftliche Vorteile, aber nicht für alle. Kritikerinnen und Kritiker der Gentechnik betonen, dass kleinere und familiär geführte Höfe, Betriebe in Subsistenzwirtschaft oder solche in kleinräumigen Agrarlandschaften kaum oder gar nicht von gentechnisch veränderten Pflanzen profitieren. Der Einsatz der Gentechnik begünstig nach dieser Sichtweise lediglich große Agrarfarmen mit starker Ausprägung zu Monokulturen (IPES-Food, 2017). Neben den damit verbundenen ökologischen Risiken (siehe oben) verdrängt die zunehmend industrialisierte (und damit auch mit Gentechnik verbundene) Landwirtschaft die Existenzgrundlage der bisherigen Kultur von Klein- und Fami- 
lienbetrieben. Befürworterinnen und Befürworter der Gentechnik wenden dagegen ein, dass diese Risiken nicht von dem Einsatz der Gentechnik abhängen, sondern mit der Skalen-Ökonomie der Agrarmärkte zu tun hat. Je größer die Betriebe, desto kostengünstiger können sie produzieren und zwar weitgehend unabhängig von der Produktionsweise. Gentechnik könne, wenn entsprechend ausgeprägt, auch und gerade für Kleinbäuerinnen und Kleinbauern wichtige Vorteile bieten (Zaidi et al., 2019; Montenegrode, 2020).

d. Aus wirtschaftlichen Gründen, so die Skeptikerinnen und Skeptiker, sei eher eine Einengung des Angebots an gentechnisch veränderten Pflanzensorten auf einige wenige weltweit einsatzfähige Nutzpflanzen zu erwarten als eine Differenzierung nach kleinräumigen Anforderungen. Dadurch könnte auch die Artenvielfalt weiter dezimiert und der Weg in eine nachhaltige Kreislaufwirtschaft verbaut werden (Morawicki/Diaz Gonzales, 2018). Befürworterinnen und Befürworter der Gentechnik halten diesem Argument entgegen, dass es gerade mit dem Einsatz der Gentechnik, vor allem der CRISPR/Cas-Technologie möglich sei, regional angepasste Nutzpflanzen zu erzeugen (Zhang et al., 2020). Ein Markt für regionale Produkte sei durchaus für die Industrie attraktiv und werde auch bereits genutzt. Vor allem ermögliche die Gentechnik, Nutzpflanzen an die zu erwartenden Klimaänderungen und die neuen Siedlungsstrukturen von Stadt und Land anzupassen (Islam et al., 2020: 1680). Zudem gebe es weder einen Zwang zur Monofunktionalisierung (die Ausrichtung von Kulturpflanzen auf eine einzige zu optimierende Kenngröße, wie etwa Ertrag pro Hektar), noch ginge diese Entwicklung von der Gentechnik aus.

e. Auskreuzungen mit gentechnisch veränderten Organismen können insbesondere für ökologisch wirtschaftende Landwirtinnen und Landwirte, aber auch für Imkerinnen und Imker zu ökonomischen Einbußen bis hin zur Existenzbedrohung führen (Braun et al., 2013: 36). Viele Kritikerinnen und Kritiker sind skeptisch, ob die rechtlich vorgeschriebenen Mindestabstände und die Vorgaben zur fachlichen Praxis ausreichen, um die Koexistenz von konventionellem oder ökologischem auf der einen und durch transgene Nutzpflanzen geprägtem Landbau auf der anderen Seite sicherzustellen.

Fazit: Unbestritten hat Gentechnik das Potenzial, mittel- und langfristige wirtschaftliche Vorteile für Anbieter von Agrardienstleistungen, Saatguthersteller und landwirtschaftliche Betriebe zu bieten. Umstritten ist, wem diese Vorteile überwiegend zugutekommen. Dabei ist zu beachten, dass viele Einkommens- und Struktureffekte durch andere Faktoren stärker beeinflusst werden und der Einsatz der Gentechnik hier nur eine geringe Rolle spielt. In welche Richtung sich die (überschaubaren) Wirkungen der 
Gentechnik entwickeln, hängt weitgehend von den Rahmenbedingungen des Gentechnikeinsatzes ab (EASAC, 2013). So haben z. B. genossenschaftlich organisierte Kleinbetriebe in Indien eigene Labore für gentechnisch modifizierte Pflanzen gegründet, um die eigene Existenzfähigkeit zu verbessern (Kuruganti, 2009). Umgekehrt können große Agrarfarmen mithilfe gentechnisch veränderter Pflanzen effizienter wirtschaften und kleinere Betriebe aus dem Markt drängen (Busch et al., 2002: 72). Ob das ohne Gentechnik anders verlaufen würde, ist dabei allerdings fraglich. Gentechnik kann im laufenden Konzentrationsprozess kaum als Verursacher, aber durchaus als Promotor dieser Entwicklung angesehen werden. Bei der Frage der Einkommenssicherung auch für kleinere Betriebe ist die Situation analog einzuschätzen. Theoretisch kann der Einsatz der Gentechnik auch das Einkommen von Kleinbetrieben verbessern und auf Dauer sicherstellen, aber unter den gegebenen Marktbedingungen ist diese Chance selten verwirklicht. Das muss aber in Zukunft nicht so weiterbestehen, wenn entsprechende Änderungen in der Marktstrategie der Anbieterfirmen vorgenommen werden oder neue Regulierungen in Kraft treten würden. Zurzeit kommen aber die Vorteile der Gentechnik überwiegend den großen und kapitalkräftigen Landwirtschaftsbetrieben zugute.

\subsubsection{Die soziale Komponente der Nachhaltigkeit}

Mit Blick auf die soziale Komponente der Nachhaltigkeit lassen sich vier Ziele für den Bereich der Gentechnik ausmachen: Gleichheit der Lebensbedingungen (intra- und intergenerationale Gerechtigkeit), Erhalt der menschlichen Gesundheit, Souveränität und Teilhabe am wirtschaftlichen und politischen Geschehen sowie soziale und kulturelle Akzeptanz (Opielka/Peter, 2018: 12 ff.; Renn et al., 2021). Ähnlich wie bei der ökologischen und der ökonomischen Komponente lassen sich hier wieder Chancen und Risiken der Gentechnik aufzeigen. Zunächst die Chancen:

a. Die Gewährleistung der inter- und intragenerationalen Gerechtigkeit ist das Herzstück der Nachhaltigkeit. In fast allen Definitionen und Erläuterungen zur Nachhaltigkeit spielt der Gedanke der gerechten Teilhabe der Menschen an den natürlichen und wirtschaftlich erzeugten Ressourcen eine zentrale Rolle. Inwieweit die Anwendungen der Gentechnik auf diese zentrale Größe Einfluss nehmen, ist in der Literatur umstritten (Knöpffler, 2018: 118). Die Befürworterinnen und Befürworter betonen, dass mit dem Einsatz der Gentechnik mengenmäßig ausreichende und auch für ärmere Bevölkerungsschichten bezahlbare Nahrungsmittel verfügbar werden. Auch kleinere landwirtschaftliche Betriebe könnten langfristig von einer engen Kooperation mit Saatgutherstellern und anderen Zulieferindustrien profitieren, denn gerade kleinere und einkommensschwache Betriebe in den Entwicklungsländern 
(und nicht nur die Großgrundbesitzerinnen und Großgrundbesitzer) könnten mit den gentechnisch veränderten Sorten mehr Einkommen, vor allem aber langfristige Einkommenssicherheit, erzielen (Brookes/Barfoot, 2016). Die Kritikerinnen und Kritiker der Gentechnik bestreiten diese Vorteile (Gottwald, 2011).

b. Was die Gesundheit anbetrifft, können gentechnische Anwendungen, vor allem wenn sie bestimmte Nährstoffe in Pflanzen anreichern helfen, positive Gesundheitsauswirkungen mit sich bringen (Islam et al., 2020: 1679). Bestes Beispiel ist die Erhöhung der Vitamin-A-Konzentration in der Reispflanze, um damit Vitaminmangel auszugleichen (Buiatti et al., 2013; Giuliano, 2017). Solche positiven gesundheitlichen Effekte sind mit der Ausweitung der CRISPR/Cas-Technik in Zukunft noch häufiger zu erwarten (Gao, 2018).

Bei den beiden Unterpunkten Teilhabe und Akzeptanz gibt es keine positiven Effekte, allerdings weisen vor allem die Befürworterinnen und Befürworter darauf hin, dass die Risiken, die sich in diesen beiden Punkten ergeben, nicht von der Gentechnik per se ausgehen, sondern von der Art ihrer Einführung und Regulierung abhängen. Dagegen betonen die Gegnerinnen und Gegner vor allem die folgenden Risiken:

a. Wie schon bei den wirtschaftlichen Auswirkungen beschrieben, begünstigen gentechnische Verfahren vor allem Großbetriebe und agro-industrielle Kooperationen, etwa zwischen Saatgutherstellern, Chemieunternehmen, größeren Betrieben und der Nahrungsmittelindustrie. Dabei bleiben kleinere und auf lokale Märkte orientierte Betriebe außen vor und sind nicht mehr konkurrenzfähig (IPES-Food, 2017; Gottwald, 2018). Die Konzentration auf große Agrarbetriebe nimmt weltweit zu und damit erhöht sich die Wahrscheinlichkeit einer ungerechten Verteilung von Ressourcen auf die jetzige und die kommende Generation. All dies ist nicht zwangsläufig mit dem Einsatz von Gentechnik verbunden, sondern liegt im globalen Trend mit und ohne den Einsatz der Gentechnik. Allerdings können gentechnische Anwendungen als Beschleuniger und Unterstützer dieses Trends interpretiert werden.

b. Viele Kritikerinnen und Kritiker der Gentechnik befürchten, dass gentechnisch verändertes Saatgut die bestehende Abhängigkeit der Landwirtinnen und Landwirte von wenigen Großfirmen verstärken könnte (Morawicki/Diaz Gonzales, 2018). Da es sich bei gentechnisch verändertem Saatgut fast ausschließlich um Hybridsorten handelt, sind die Landwirtinnen und Landwirte darauf angewiesen, immer wieder neues Saatgut bei dem jeweiligen Hersteller zu kaufen. Zudem werden von multinationalen Unternehmen Patente an Nutzpflanzen erworben, durch die sie ihre Macht gegenüber Konkurrenten und Abnehmern ausbauen können (Busch et al., 2002: 72). Viele Beobachterinnen und Beobachter sind daher besorgt, dass vor allem die klei- 
nen, oft auf Subsistenz hin ausgerichteten Kleinbetriebe in den Entwicklungsländern in zunehmende Abhängigkeiten geraten, weil sie keine freie Wahl mehr haben $\mathrm{zu}$ entscheiden, welches Saatgut sie kaufen wollen und wie sie ihre traditionellen Rechte wahren können. Inzwischen bieten einzelne Firmen auch Gesamtpakete an (etwa Herbizide mit herbizidresistentem Saatgut), die langfristige Abhängigkeiten zementieren können. ${ }^{3}$ Insofern besteht das Risiko, dass die Abhängigkeiten zunehmen und die Handlungsfreiheit der in der Landwirtschaft tätigen Personen weiter abgebaut wird.

c. Die Ablehnung der Gentechnik war zu Beginn der Debatte stark von Sorgen um gesundheitliche Nebenwirkungen gentechnisch veränderter Pflanzen geprägt. Bei der Gentechnik der ersten und zum Teil der zweiten Generation wurden häufig Botenstoffe oder Marker eingesetzt, die auf die Resistenz der Pflanzen Einfluss nehmen (Promotoren, Trailor etc.). Diese Resistenzen, etwa gegen Herbizide, können sich auf andere Pflanzen (etwa Unkräuter) übertragen oder auch bei den Konsumentinnen und Konsumenten der Pflanzen (Tiere und Menschen) Probleme erzeugen (so wurden etwa in den frühen Jahren der Gentechnikanwendung Antibiotika eingesetzt). Inzwischen sind mit CRISPR/Cas diese Nebenwirkungen auf ein Minimum reduziert (Metha et al., 2020). Kritikerinnen und Kritiker der Gentechnik weisen aber weiterhin auf mögliche, nicht erkannte Langzeiteffekte gentechnisch veränderter Pflanzen hin und halten das Risiko von gesundheitlichen Langzeitfolgen für noch nicht ausreichend geklärt (Cellini et al., 2004; Catak/Atalay, 2020).

d. Durch die Aufnahme von fremden Gensegmenten können beim Menschen allergische Reaktionen ausgelöst werden, wenn eine Unverträglichkeit besteht (Islam et al., 2020: 1682). Kritikerinnen und Kritiker befürchten, dass bei Konsumentinnen und Konsumenten über die Herkunft der Fremdgene keine Kenntnis herrsche, wodurch allergische Reaktionen nicht mehr vorhersehbar werden. ${ }^{4}$ Befürworterinnen und Befürworter der Gentechnik sehen dagegen keinen Grund zur Sorge: Bei fast allen als Fremdgene eingesetzten Arten liegen nach den Erfahrungen der medizinischen Praxis keine Fälle von allergischen Reaktionen vor. Außerdem könne man dieses Problem durch eine entsprechende Kennzeichnung regeln (Worm et al., 2021).

3 „Gegner der Grünen Gentechnik verknüpfen die Anwendung von GV-Pflanzen oft mit der Abhängigkeit von Bauern von einigen wenigen Saatgutfirmen, die nebst Patentrechten für das Saatgut auch die Patentrechte für die zugehörigen Pflanzenschutzmittel besitzen. Die Stärkung der Agrarforschung an öffentlichen Institutionen und der Wissenstransfer in Entwicklungsländer könnten dieser Entwicklung entgegenwirken“" (Braun et al., 2013: 50).

4 „Was die menschliche Gesundheit betrifft, so ist die größte Befürchtung die Schaffung neuer Allergene und der Gentransfer von GVO-Lebensmitteln auf menschliche Zellen oder die Darmmikroflora“ (Klümper/Qaim, 2014; Übersetzung durch den Verfasser). 
Allerdings ist zu beachten, dass bei einer starken Ausweitung des Einbaus von fremden Gensegmenten Allergikerinnen und Allergiker die Übersicht verlieren, welche potenziell allergenen Stoffe in welchen Lebensmitteln vorhanden sind. Auch hier ändert sich durch die Einführung von neuen gentechnischen Verfahren, die nicht mehr auf den Einbau von Fremdgenen angewiesen sind, die Ausgangssituation, allerdings bleibt das Risiko prinzipiell bestehen. Ähnliche Argumente gelten auch für Futtermittel (Snell et al., 2012).

e. Zweifellos bestehen bei der Gentechnik erhebliche Akzeptanzprobleme (Peters et al., 2007; Renn, 2018; zu einer aktuellen Befragung der deutschen Bevölkerung siehe Hampel et al., Kap. 24; scharfe Kritik an der geringen Akzeptanz der Gentechnik in Europa üben van den Daele/Broer, Kap. 21). Diese sind in Europa besonders stark ausgeprägt, aber Bedenken gegen Gentechnik bis hin zu Protesten und Verwüstungen von Feldern mit gentechnisch veränderten Pflanzen finden sich auch in Asien, den USA und selbst einigen Schwellen- und Entwicklungsländern (Sikora/Rzymski, 2021). Dabei ist der Protest weitgehend durch die Wahrnehmung eines mangelnden Nutzens für Verbraucherinnen und Verbraucher, die Sorge um eine weitere Industrialisierung der Landwirtschaft und die Ablehnung einer von Konzernen bestimmten Form der Landnutzung geprägt (Renn, 2007). Mangelnde Akzeptanz hat auch prägenden Einfluss auf die Regulierung von gentechnisch veränderten Pflanzen und Tieren, wie z. B. in der Novel Food Verordnung der EU. Denn Politikerinnen und Politiker reagieren auf die zum Teil gut organisierten Gruppen, die der Gentechnik kritisch gegenüberstehen, und versuchen, über strenge Regulierungen die öffentliche Meinung zur grünen Gentechnik positiv zu beeinflussen.

f. Indirekt verbunden mit den Akzeptanzproblemen sind rechtliche und finanzielle Risiken, die sich aus den bestehenden gesetzlichen Regelungen und Regulierungen ergeben (Bernauer et al., 2011). Finanzielle Haftungen sind häufig nicht auf nachweisbare Schäden (etwa auf die Gesundheit) bezogen, sondern sind mit der Nichteinhaltung von Reinheitsgeboten (die z. B. durch bestimmte Anteile gentechnisch veränderter Produkte in Nahrungsmitteln festgelegt sind) oder der Verletzung von Abstands- und Zulassungsbestimmungen verbunden (Busch et al., 2002: 76 ff.).

Fazit: Gerade im Bereich der sozialen Nachhaltigkeit sind die Potenziale für Chancen eher gering, dagegen die Risiken besonders umfassend, aber nicht unbedingt schwerwiegend. Mehr noch als im Bereich der ökologischen und ökonomischen Nachhaltigkeit sind die Chancen und Risiken oft zwei Seiten derselben Medaille. So führen die erhöhten Erträge verbunden mit geringeren Kosten für Pestizide und Herbizide zu mehr Einkommen und betriebswirtschaftlicher Konkurrenzfähigkeit landwirtschaftlicher 
Betriebe, aber dies kann gerade Konzentrationsprozesse, die Kleinbetriebe zur Vergrößerung oder Aufgabe zwingen, fördern und die Abhängigkeit der kleinen Betriebe von den mächtigen Partnern der Wertschöpfungskette vergrößern. Die Funktionalisierung der Nahrungsmittelproduktion, die durch die Anwendungen der Gentechnik unterstützt wird, verspricht mehr Effizienz, trägt aber gleichzeitig zu einer Entfremdung der Konsumentinnen und Konsumenten von oft romantisierenden Leitbildern einer bäuerlichen Landwirtschaft bei (siehe Hampel et al., Kap. 24). In den Augen vieler Betrachterinnen und Betrachter reiht sich die Gentechnik in eine Assoziationskette von maximaler Profitausrichtung, industrialisierten Praktiken, inhumaner Tierhaltung und wirtschaftlichen Konzentrationsprozessen ein (Renn, 2018). All das ist nicht zwangsläufig mit dem Einsatz der Gentechnik verbunden, wird aber in den mentalen Modellen einer Großzahl von Menschen besonders in Europa so wahrgenommen (Sikora/Rzymski, 2021: 298). Nach wie vor ist die geringe Akzeptanz verbunden mit entsprechend strikten staatlichen Regulierungen. Mehr und mehr stehen nicht mehr die nachweisbaren oder auch durch Evidenz nachgewiesenen Folgen für Ökologie, Wirtschaft und Sozialwesen im Vordergrund, sondern vielmehr die Einhaltung von Regelungen ohne Bezug zu einem nachweisbaren Schaden (Callaway, 2018).

\subsection{Aussichten}

Mit der landwirtschaftlichen Gentechnik sind zahlreiche offene Fragen um Folgewirkungen für Natur und Artenvielfalt, Mensch und Organismus verbunden. Viele dieser Fragen betreffen Chancen und Risiken vor allem im Hinblick auf die Ziele der nachhaltigen Entwicklung. Ob sich Chancen und Risiken in ihrer Qualität von den Folgewirkungen der herkömmlichen Pflanzenzüchtung unterscheiden, ist strittig. Gentechnische Anwendungen besitzen aber das Potenzial, die Entwicklung sowohl der Chancen wie der Risiken zu beschleunigen oder im jeweiligen Ausmaß zu verstärken. Gleichzeitig sind mit dem Einsatz gentechnisch veränderter Pflanzen Erwartungen verbunden, zu dem Ziel einer ausreichenden und qualitativ hochwertigen Ernährung der Bevölkerung beitragen zu können (Islam et al., 2020). Ernährungskrisen haben sich allerdings häufiger als Folge verfehlter Landwirtschafts- und Verteilungspolitik herausgestellt. Sie ergeben sich oft als Konsequenz der mangelnden Kaufkraft, also der weltweiten Armut. Politisches und wirtschaftliches Fehlverhalten kann aber auch durch verbesserte gentechnische Verfahren und Produkte nicht ausgeglichen werden. Gleichzeitig kann die Gentechnik nicht als Sündenbock für alles, was in der globalen Landnutzung problematisch verläuft, verantwortlich gemacht werden. 
Die Zukunft der Gentechnik hängt stark vom Gestaltungswillen der beteiligten Akteurinnen und Akteure ab. ${ }^{5}$ Wenn man die drei Komponenten der Nachhaltigkeit als Bewertungskriterien zu Grunde legt, wird deutlich, dass die Gentechnik wesentliche Potenziale für eine auf Nachhaltigkeit orientierte Transformation der Gesellschaft bietet. Gleichzeitig sind aber mit der Gentechnik viele zum Teil übergreifende Risiken verbunden, die aber durch eine sachlich fundierte und von Werten des Gemeinwohls getragene Gestaltung zumindest eingeschränkt, wenn nicht sogar vermieden werden können (Dürnberger, 2019: 68ff.). Die Analyse der verschiedenen Auswirkungen auf die drei Komponenten der Nachhaltigkeit zeigt auf, dass die negativen Konsequenzen nicht zwangsläufig mit dem Einsatz der Gentechnik verbunden sind und sich die positiven Folgen auch nur dann durchsetzen werden, wenn sie aktiv gewollt in die Geschäftspolitik der Unternehmen, aber auch in die Regulierungspolitik der Regierungen einbezogen werden. Eine der Nachhaltigkeit verpflichtete Anwendungsstrategie für gentechnisch modifizierte Pflanzen (und auch Tiere) könnte neue Impulse für eine weitere öffentliche Debatte um die Vorteile und Nachteile der Gentechnik setzen. ${ }^{6}$

Die Zeit für eine Neubesinnung ist gerade günstig. Die Gentechnik hat als Streitthema in den letzten drei, vier Jahren in der Öffentlichkeit an Schärfe verloren (Albrecht

5 „Rechenschaftspflicht kann auch in Form von Forschungsmanagement oder wissenschaftspolitischen Organisationen erfolgen, die sich direkt an Prozessen der gemeinschaftlichen Überprüfung mit Landwirten, Verbrauchern und Arbeitern entlang der gesamten Lieferkette, in denen die gentechnischen Technologien ihre Spuren hinterlassen, unter realen Kontextbedingungen beteiligen. Anstatt als Strafmaßnahme gesehen zu werden, ermöglicht uns die Rechenschaftspflicht, den Fortschritt - oder das Fehlen davon - bei den oben genannten Elementen zu überwachen, was letztendlich ein strengerer Ansatz für die Technologiebewertung bedeutet, aber dafür auch den Weg für die Schaffung öffentlichen Vertrauens vorbereitet" (Montenegrode, 2020; Übersetzung durch den Verfasser).

6 „Wir schlagen vor, dass einige Bedenken in einem Dialog gütlich ausgehandelt werden können. Deontologische Argumente wie die Natürlichkeit sind schwieriger zu verhandeln, da sie sich auf tief empfundene ethische oder kulturelle Werte beziehen. Ein Schritt nach vorne wäre es aber, wenn auch für diesen Argumentationsstrang die Teilnehmerinnen und Teilnehmer am Dialog informierte Entscheidungen treffen könnten und gleichzeitig diese Entscheidungen in einer respektvollen Weise koexistieren können. Innerhalb des Kontextes sozialer Bewegungen können gentechnisch veränderte Sorten auch für bestimmte Funktionen gut passen oder begrüßt werden, sofern sie Eigenschaften enthalten, die zu erfolgreichen IPM-Programmen beitragen und sozial verträglich sind. [...] Zum Beispiel können Machtfragen rund um den Einsatz von Gentechnik schwierig zu lösen sein, da sie Teil einer komplexen gesellschaftlichen Debatte über die Verteilung von Erträgen zwischen allen Beteiligten (Züchter, Landwirte, Handel, Verbraucher) sind und auch mit Diskussionen über Monopole zu tun haben. Es können jedoch institutionelle Vereinbarungen getroffen werden, um solche Machtfragen konstruktiv anzugehen. Es gibt Wettbewerbsgesetze, die unerwünschte Monopole verhindern oder aufbrechen können. Gemeinsame Vereinbarungen können in der Diskussion zwischen den Interessengruppen entwickelt werden [...] “" (Lotz et al., 2020: 27f.; Übersetzung durch den Verfasser). 
et al., 2017). Mit CRISPR/Cas kommt eine neue Debatte auf, die von den gentechnikkritischen NGOs wie Greenpeace inzwischen differenzierter als früher aufgegriffen wird. Mein Eindruck ist, dass der Protest insgesamt etwas verhaltener ausfällt, weil auch die Kritikerinnen und Kritiker der Gentechnik Fortschritte in der Berücksichtigung ihrer Anliegen wahrnehmen. Nach wie vor gibt es aber gegen Anwendungen in der industriellen Landwirtschaft große Vorbehalte, vor allem in den Umweltverbänden. Hier wird mit Argwohn beobachtet, ob man das Alte mit neuen Namen wie Bioökonomie und Biotechnologie erneut salonfähig machen will. Zwar ist die Auseinandersetzung nicht mehr so verbissen wie vor zehn Jahren, aber bei den NGOs bleibt das Thema sensibel.

Die Themen der Auseinandersetzung haben sich auch im Verlauf der Debatte geändert. Am Anfang stand der Gesundheitsschutz im Vordergrund, also die Frage, ob gentechnisch veränderte Lebensmittel die menschliche Gesundheit mittel- und langfristig gefährden können. Heute stehen andere Anliegen im Vordergrund: die industrialisierte Landwirtschaft, die Abhängigkeit von Großkonzernen, die zunehmende Industrialisierung der Nahrungskette, langfristige ökologische Veränderungen sowie die Beschränkung (und damit Reduzierung) der Kulturpflanzen auf wenige gentechnisch veränderte Sorten (Islam et al., 2020). Auch wenn die Themen sich wandeln, bleiben die Leitbilder, die hinter Zustimmung und Ablehnung stehen, relativ konstant und unbeweglich (Renn, 2018; Gottwald, 2018: 101 f.). Zum einen findet man die Vision einer naturnahen Landwirtschaft, getragen von kleinen ländlichen Betrieben, die sich und die umgebende Bevölkerung ausreichend ernähren können und dabei gleichzeitig auf ökologische Werte, wie Erhalt der Biodiversität und hohe Bodenqualität, achten. Ziel ist die Selbstversorgung in einer Region, weniger der Export. Dieser Entwurf funktioniert global nur, wenn wir die heutigen Ernährungsgewohnheiten von Fleisch auf mehr pflanzliche Kost umstellen und auch höhere Preise für Lebensmittel als akzeptabel einstufen.

Bei dem Gegenentwurf ist die Landwirtschaft ein Wirtschaftsfaktor wie jeder andere, mit hohem Modernisierungspotenzial auf der Basis von Digitalisierung und Gentechnik. Es geht um mehr Effizienz und Erhöhung der Erträge, weil immer weniger Boden zur Verfügung steht. Wegen der weiter anwachsenden Weltbevölkerung muss in dieser Vision die Produktivität enorm gesteigert werden. Dieser Entwurf würde eine Änderung unserer Ernährung nicht erforderlich machen und auch einen hohen Fleischbedarf in den sich entwickelnden Ländern verkraften. Gentechnik ist dabei nur ein Baustein unter vielen: Letztendlich stehen am Ende dieser Entwicklung der Modernisierung einige wenige global agierende Agrarkonzerne, die weltumspannend die Bo- 
denbewirtschaftung für Nahrungsmittel oder Biostoffe mithilfe modernster Technik und wissenschaftlich geprüfter Optimierung übernehmen. ${ }^{7}$

Aufbauend auf diesen beiden gegensätzlichen Visionen, die jeweils Endpunkte eines breiten Spektrums an Visionen widerspiegeln, könnte der Rekurs auf Nachhaltigkeit eine Form des Dialogs zwischen den Fronten ermöglichen (Acatech, 2012; One Planet Network, 2020: 22). Wie oben angedeutet, ist die Gentechnik weder per se nachhaltig noch nicht-nachhaltig. Entscheidend ist die Frage, wie die Anwendungen der Gentechnik ausgestaltet werden, sodass sie den Zielen der Nachhaltigkeit dienen können. Unbestritten ist, dass die Menschheit die Ressourcen der Erde nicht übernutzen darf und die Emissionen und Abfälle reduzieren bzw. in die Kreislaufwirtschaft integrieren muss (Kellermann, 2020b). Dazu kann, bei entsprechenden Rahmenbedingungen und Zielsetzungen, die Gentechnik einen Beitrag leisten.

Zu den ökologischen Zielen der Nachhaltigkeit treten noch die ökonomischen und die sozialen Aspekte. Beide spielen bei der Debatte um Gentechnik zurzeit noch eine untergeordnete Rolle, obwohl gerade die Frage nach sozialer Gerechtigkeit und Autonomie mit der Praxis von Gentechnik eng verbunden ist. Nicht zuletzt geht es um die Struktur der Landwirtschaft. Gentechnik kann Monokulturen unterstützen, aber auch die Vielfalt an Lebensmitteln erweitern. Im Moment wird Gentechnik eher dazu eingesetzt, die Zahl der für Lebensmittel eingesetzten Pflanzensorten zu reduzieren. Das ist eine Frage der Prioritätensetzung, nicht der Methodik.

Damit Nachhaltigkeit als Leitidee für den Einsatz der Gentechnik auch faktisch umgesetzt wird, sind neue kooperative Formen der Verständigung zwischen Industrie, Landwirtinnen und Landwirten, Umweltschutzorganisationen sowie politischen Regulierungsinstanzen notwendig. Dabei muss das Thema Gentechnik in einen größeren Zusammenhang mit Landnutzung, Treibhausgasemissionen und Ernährungsverhalten eingebettet werden (Butkowski et al., 2020). Die Einrichtung solcher kooperativer Netzwerke und Plattformen könnte dazu führen, Treiber und Hemmnisse landwirtschaftlicher Praktiken systemisch zu beleuchten, vor allem unter der Randbedingung der Digitalisierung, der neuen Entwicklungen in der Gentechnik (CRISPR/Cas) und der Forderung nach nachhaltiger Entwicklung (Clément/Ajena, 2021). Wenn man diese größeren Themen miteinander verzahnt, ist die Gentechnik ein Mosaikstein unter vielen. Das könnte auch dazu beitragen, dass Befürworterinnen und Befürworter sowie Kriti-

7 „Beispielsweise könnte bei [...] partizipativen Modellen angesichts zukünftiger Technikkontroversen stärker darauf geachtet werden, von Beginn an nicht allein auf die Potenziale und Risiken der zu behandelnden Technologie zu fokussieren, sondern darüber hinaus bewusst auch Subkonflikte und Vorstellungswelten, Metaphern und Narrationen in der Diskussion zuzulassen sowie zum Thema zu machen“ (Dürnberger, 2019: 251). 
kerinnen und Kritiker der Gentechnik ihr Gesicht wahren können. Das heißt nicht, dass man im Rahmen dieser Netzwerke nicht auch über Gentechnik und ihre Möglichkeiten und Grenzen debattieren sollte, aber dieses Thema ist in einem größeren Rahmen von neuen Entwicklungen, Transformationen und Überschneidungen mit anderen Feldern wie Energie und Süßwassernutzung eingebunden (Gottwald, 2011). Mit einem Ansatz wie „neue Leitbilder für die Landwirtschaft und Landnutzung der Zukunft" wäre viel mehr gewonnen als mit einem engen Dialog zum Thema Gentechnik. Dann kämen Themen wie Methoden der Saatgutverbesserung, ob über Züchtung, Gentechnik oder andere Methoden, oder Intensivierung der Landnutzung von selbst zur Sprache (Godfray, 2015: 205). Auch die Anpassung an den Klimawandel wäre ein wesentlicher Gesichtspunkt, z. B. welche Rolle gentechnisch veränderte Pflanzen bei der Strategie der Anpassung (,adaptation“) an die Auswirkungen des jetzt schon eingetretenen Klimawandels spielen könnten. Mit solchen Dialogen über Zusammenhänge kann man Themen wie Gesundheit, Landwirtschaft und Ernährung ohne ideologische Scheuklappen zusammenbringen und die Implikationen der unterschiedlichen Leitbilder für Landwirtschaft auf der Basis der oben aufgezeigten Kriterien für Nachhaltigkeit überprüfen.

Im Rahmen sozialer Bewegungen ist Gentechnik häufig zur Mobilisierung von Protest und Akzeptanzverweigerung eingesetzt worden. Dabei übernimmt die Debatte um ihre Risiken und Probleme häufig eine Stellvertreterfunktion für die grundlegende Fragestellung, ob ein weiteres Voranschreiten der Landwirtschaft in Richtung auf industrielle Verwertungseffizienz und Verwertungsfunktionalität wünschenswert sei. Um die Chancen der Gentechnik sinnvoll zu nutzen, die Risiken effektiv begrenzen und verantwortbar mit dieser neuen Technologie umgehen zu können, muss der gesellschaftliche Diskurs über die Zukunft der Landwirtschaft verstärkt und die Nutzung der Gentechnik erkennbar in einen von Kriterien der Nachhaltigkeit geprägten Gestaltungsauftrag eingebunden werden.

\subsection{Literaturverzeichnis}

Acatech (2012): Perspektiven der Biotechnologie-Kommunikation. Kontroversen - Randbedingungen - Formate. (Acatech Position). Springer Verlag, Heidelberg.

Akademien der Wissenschaften Schweiz (2013): Gentechnisch veränderte Nutzpflanzen und ihre Bedeutung für eine nachhaltige Landwirtschaft in der Schweiz. Akademien der Wissenschaften der Schweiz, Bern.

Albrecht, S. et al. (2017): Green genetic engineering and genome editing. In: TATuP - Zeitschrift für Technikfolgenabschätzung in Theorie und Praxis 26(3): 64-69, Online-Publikation 18.12.2017. DOI: 10.14512/tatup.26.3.64.

Altieri, M. A. (2018): Agroecology: The science of sustainable agriculture. CRC Press, Boca Raton. 
Bailey-Serres, J. et al. (2019): Genetic strategies for improving crop yields. In: Nature 575(7781): 109118.

Benbrook, C. (2016): Trends in glyphosate herbicide use in the United States and globally. In: Environmental Science Europe 28(3), Online-Publikation 02.02.2016. DOI: 10.1186/s12302-016-0070-0.

Benighaus, C./Renn, O. (2009): Das Mediationsverfahren zur Anwendung in der grünen Gentechnik. In: Göpfert, J./Moos, T. (Hrsg.): Konfliktfelder beackern. Dialog- und Partizipationsverfahren bei fundamentalen Technikkonflikten am Beispiel der Grünen Gentechnik. VS Verlag für Sozialwissenschaften, Wiesbaden: 65-92.

Bernauer, T. et al. (2011): Government regulation and public opposition create high additional costs for field trials with GM crops in Switzerland. In: Transgenic Research 20:1227-1234.

Braun, R. et al. (2013): Gentechnisch veränderte Nutzpflanzen und ihre Bedeutung für eine nachhaltige Landwirtschaft in der Schweiz. Akademien der Wissenschaften Schweiz, Bern.

Breeman, G. et al. (2017): Food security and the sustainability of GMOs in the United States and the European Union. In: Barling, D. (Hrsg.): Advances in food security and sustainability. Elsevier, New York: 165-193. DOI: 10.1016/bs.af2s.2017.09.005.

Brookes, G./Barfoot, P. (2016): Global income and production impacts of using GM crop technology 1996-2014. In: GM Crops Food 7: 38-77.

Buiatti, M. et al. (2013): The application of GMOs in agriculture and in food production for a better nutrition: two different scientific points of view. In: Genes Nutrion 8: 255-270.

Busch, R. J. et al. (2002): Grüne Gentechnik - ein Bewertungsmodell. Utzverlag, München.

Butkowski, O. K. et al. (2020): Examining the social acceptance of genetically modified bioenergy in Germany: Labels, information valence, corporate actors, and consumer decisions. In: Energy Research \& Social Science 60, Online-Publikation 16.10.2019. DOI: 10.1016/j.erss.2019.101308.

Callaway, E. (2018): CRISPR plants now subject to tough GM laws in European Union. In: Nature 560(7716): 16-17.

Carpenter, J. E. (2011): Impact of GM crops on biodiversity. In: GM Crops 2: 7-23.

Catak, E./Atalay, A. (2020): Review: Effects of GMO agricultural products on living things. In: Journal of Botanical Research, 3: 16-19. DOI: 10.30564/jrb.v2i1.1764.

Cellini, F. et al. (2004): Unintended effects and their detection in genetically modified crops. In: Food and Chemical Toxicology 42: 1089-1125.

Clément, C./Ajena, F. (2021): Paths of least resilience: advancing a methodology to assess the sustainability of food system innovations - the case of CRISPR. In: Agroecology and Sustainable Food Systems 45(5): 637-653. DOI: 10.1080/21683565.2021.1890307.

Coyette, B. et al. (2002): Effect of introducing glyphosate-tolerant sugar beet on pesticide usage in Europe. In: Pesticide Outlook 13: 219-223.

CSS = Critical Scientists Switzerland et al. (2019): Gene drives: A report on their science, applications, social aspects, ethics and regulations. CSS/ENSSER/VWD, Berlin/Bonn.

Dubock, A. (2019): Golden rice: To combat vitamin A deficiency for public health. In: Zepka, L. (Hrsg.): Vitamin A. Intech open Verlag, London.

Dürnberger, C. (2019): Natur als Widerspruch. Die Mensch-Natur-Beziehung in der Kontroverse um die Grüne Gentechnik. Nomos, Baden-Baden. 
EASAC $=$ European Academies Science Advisory Council (2013): Planting the future: opportunities and challenges for using crop genetic improvement technologies for sustainable agriculture. EASAC Policy Report 21. Unter: https://easac.eu/fileadmin/PDF_s/reports_statements/Planting_ the_Future/EASAC_Planting_the_Future_FULL_REPORT.pdf [07.04.2021].

Gao, C. (2018): The future of CRISPR technologies in agriculture. In: Nature Reviews. Molecular Cell Biology 19(5): 275-76. DOI: 10.1038/nrm.2018.2.

Giuliano, G. (2017): Provitamin A biofortification of crop plants: a gold rush with many miners. In: Current Opinion in Biotechnology 44: 169-180. DOI: 10.1016/j.copbio.2017.02.001[2.

Gliessman, S. R. (2015): Agroecology: The ecology of sustainable food systems. 3. Auflage. CRC press, Boca Raton.

Godfray, C. (2015): The debate over sustainable intensification. In: Food Security 7: 199-208.

Gottschlich, D. et al. (2017): Fazit zum Politikfeld Agro-Gentechnik. In: Gottschlich, D./Mölders, T. (Hrsg.): Politiken der Naturgestaltung. Springer VS, Wiesbaden: 217-224. DOI: 10.1007/978-3-65808193-5_13.

Gottwald, F.-T. (2011): Biotechnologie, Gentechnik und nachhaltige Entwicklung. In: Otto, K. S./ Speck, T. (Hrsg.): Darwin meets Business. Evolutionäre und bionische Lösungen für die Wirtschaft. Gabler, Wiesbaden: 249-258. DOI: 10.1007/978-3-8349-6381-9_22.

Gottwald, F.-T. (2018): Auf der Suche nach Regeln für eine nachhaltige Bioökonomie. Sechs Thesen zur Regulierung aus ethischer Sicht. In: Jahreschrift des DNWE (Hrsg.): Forum Wirtschaftsethik: Bioökonomie und Ethik. Deutsches Netzwerk Wirtschaftsethik, Berlin: 100-105.

IPES-Food (2016): From uniformity to diversity: A paradigm shift from industrial agriculture to diversified agroecological systems. Unter: http://www.ipes-food.org/_img/upload/files/Uniformity ToDiversity_FULL.pdf [07.04.2021].

IPES-Food (2017): Too big to feed: Exploring the impacts of mega-mergers, consolidation and concentration of power in the agri-food sector. Unter: http://www.ipes-food.org/_img/upload/files/ Concentration_FullReport.pdf [07.04.2021].

Islam, R. et al. (2020): Assessment of the effects of genetically modified (GM) foods: A brief study on health and environmental concerns. In: Journal of Materials and Environmental Sciences 11(10): 1676-1688.

James, C. (2010): A global overview of biotech (GM) crops: Adoption, impact and future prospects. In: GM Crops 1(1): 8-12.

Kellermann, K. (2020a): GVP-basierte Landwirtschaft. In: Derselbe (Hrsg.): Die Zukunft der Landwirtschaft. Konventioneller, gentechnikbasierter und ökologischer Landbau im umfassenden Vergleich. Springer Spektrum, Wiesbaden: 75-123. DOI: 10.1007/978-3-658-30359-4_4.

Kellermann, K. (2020b): Fazit. In: Derselbe (Hrsg.): Die Zukunft der Landwirtschaft. Konventioneller, gentechnikbasierter und ökologischer Landbau im umfassenden Vergleich. Springer Spektrum, Wiesbaden: 171-174. DOI: 10.1007/978-3-658-30359-4_6.

Klümper, W./Qaim, M. (2014): A meta-analysis of the impacts of genetically modified crops. In: PLoS One 9: e111629. 
Knöpffler, N. (2018): CRISPR-Methode, Nachhaltigkeit und die Grüne Gentechnik. In: Jahreschrift des DNWE (Hrsg.): Forum Wirtschaftsethik: Bioökonomie und Ethik. Deutsches Netzwerk Wirtschaftsethik, Berlin: $116-128$.

Kuruganti, K. (2009): Genetic engineering in Indian agriculture. An introductory handbook. In: New Delhi: eSocialsciences.

Lotz, L. A. et al. (2020): Genetic engineering at the heart of agroecology. In: Outlook on Agriculture 49(1): 21-28. DOI: 10.1177/0030727020907619.

Luger, O. et al. (2017): Gentechnologie in der medizinischen Anwendung am Menschen. In: Dieselben (Hrsg.): Gentechnik geht uns alle an! Ein Überblick über Praxis und Theorie. Springer VS, Wiesbaden: 115-160. DOI: 10.1007/978-3-658-15605-3_7.

Matyushenko, I. et al. (2016): Modern approaches to classification of biotechnology as a part of NBICtechnologies for bioeconomy. In: British Journal of Economics, Management \& Trade 14(4): 28151.

McCormick, K./Kautto, N. (2013): The bioeconomy in Europe: an overview. In: Sustainability 5(6): 2589-2608.

Mehta, S. et al. (2020): CRISPR/Cas9-edited rice: A new frontier for sustainable agriculture. In: Rakshit, A. et al. (Hrsg.): New frontiers in stress management for durable agriculture. Springer, Singapore: $427-58$.

Montenegrode, W. M. (2020): Democratizing CRISPR? Stories, practices, and politics of science and governance on the agricultural gene editing frontier. In: Elementa: Science of the Anthropocene 8(1): 9. DOI: 10.1525/elementa.40.

Morawicki, R. O./Diaz Gonzalez, D. J. (2018): Food sustainability in the context of human behavior. In: Yale Journal of Biological Medicine 91(2): 191-196.

Müller-Röber, M. et al. (Hrsg.) (2013): Grüne Gentechnik. Aktuelle wissenschaftliche, wirtschaftliche und gesellschaftliche Entwicklungen. Themenband der interdisziplinären Arbeitsgruppe Gentechnologiebericht. Berlin-Brandenburgische Akademie der Wissenschaften (BBAW): Forschungsberichte Interdisziplinäre Arbeitsgruppen Band 31. Forum W - Wissenschaftlicher Verlag, Dornburg.

Niggli, U. (2012): Der Prüfstein für die Gentechnologie ist die Nachhaltigkeit. In: Leitungsgruppe des NFP 59 (Hrsg.): Nutzen und Risiken der Freisetzung gentechnisch veränderter Pflanzen. Programmsynthese des NFP 59. vdf Hochschulverlag AG, Zürich: 180-183.

Nymark, M. et al. (2016): A CRISPR/Cas9 system adapted for gene editing in marine algae. In: Scientific Reports 25(6): Art. 24951.

One Planet Network (2020): Towards a Common Understanding of Sustainable Food Systems. Unter: https://www.oneplanetnetwork.org/sites/default/files/sfs_programme_glossary_towards_a_ common_understanding_of_sfs_2020.pdf [07.04.2021].

Opielka, M./Peter, S. (2018): Soziale Nachhaltigkeit der Landwirtschaft. Vergleichende Nachhaltigkeitsbewertung landwirtschaftlicher Systeme. ISÖ-Text 2018-2. Institut für Sozialökologie, Frankfurt am Main. 
Peters, H. P. et al. (2007): Culture and technical innovation, impact of institutional trust and appreciation of nature on attitudes towards food biotechnology in the USA and Germany. In: International Journal of Public Opinion Research 19(2): 191-220.

Renn, O. (2007): Grüne Gentechnik: Konfliktlinien und Möglichkeiten ihrer Überwindung. In: Köstner, B. et al. (Hrsg.): Agro-Gentechnik im ländlichen Raum - Potentiale, Konflikte, Perspektiven. J.H. Röll, Dettelbach: 41-56.

Renn, O. (2018): Gentechnik als Symbol: Zur Risikowahrnehmung der grünen Gentechnik. In: Hucho, F. et al. (Hrsg.): Vierter Gentechnologiebericht. Bilanzierung einer Hochtechnologie. Forschungsberichte der interdisziplinären Arbeitsgruppen der Berlin-Brandenburgischen Akademie der Wissenschaften Band 40. Nomos, Baden-Baden: 161-172.

Renn, O. et al. (2021): The opportunities and risks of digitalisation for sustainable development: a systemic perspective. In: GAIA 30(1): 23-28.

Rösch, C. et al. (2020): Einführung in die Nachhaltigkeit. In: Dieselben (Hrsg.): Bioökonomie im Selbststudium: Nachhaltigkeit und ökologische Bewertung. Zertifikatskurs Bioökonomie. Springer Spektrum, Berlin: 1-11. DOI: 10.1007/978-3-662-61383-2_1.

Scholz, R. W. et al. (2021): Vulnerabilität und Stützung der globalen Ernährungssicherheit durch digitale Daten: Globale Ernährungssicherheit. In: Scholz, R. W. et al. (Hrsg.): Supplementarische Information zum DiDaT Weissbuch: Orientierungen verantwortungsvollen Umgang mit Daten Orientierungen eines transdisziplinären Prozesses. Nomos, Baden-Baden: 183-193.

Sikora, D./Rzymski, P. (2021): Public acceptance of GM foods: A global perspective (1999-2019). In: Singh, P. et al. (Hrsg.): Policy issues in genetically modified crops: Global perspective. Academic Press, London: 293-315.

Snell, C. et al. (2012): Assessment of the health impact of GM plant diets in long-term and multigenerational animal feeding trials: A literature review. In: Food and Chemical Toxicology 50: 1134-1148.

Steinhoff, C. (2005): Der aktuelle Begriff: Grüne, Rote, Weiße und Graue Gentechnik. Wissenschaftliche Dienste des Deutschen Bundestages (18/05). Unter: https://www.bundestag.de/resource/blob/ 513504/26d3241d5107107b47b3cae35d47f428/gruene--rote--weisse-und-graue-gentechnik-data.pdf [07.04.2021].

Tang, G. et al. (2009): Golden rice is an effective source of vitamin A. In: The American Journal of Clinical Nutrition 89(6): 1776-1783.

UNEP (2019): Collaborative framework for food systems transformation: A multi-stakeholder pathway for sustainable food systems. Unter: https://www.oneplanetnetwork.org/sites/default/ files/un-e_collaborative_framework_for_food_systems_transformation_final.pdf [07.04.2021].

UN = United Nations (2019): Global Sustainable Development Report. In: The future is now - science for achieving sustainable development. United Nations, New York.

Vogt, M./Hagemann, H. (2010): Zwischen Ernährungssouveränität, Exportorientierung und Energiegewinnung. Sozialethische Analysen zu Landwirtschaft und Ernährungssituation in Afrika. In: Amos International 4: 19 -27. 
Wolter, F. et al. (2019): Plant breeding at the speed of light: The power of CRISPR/Cas to generate directed genetic diversity at multiple sites. In: BMC Plant Biology 19(1): 176. DOI: 10.1186/s12870019-1775-1.

Worm, M. et al. (2021): Nahrungsmittelallergien - ein Überblick. In: HNO Nachrichten 51: 22-27. DOI: 10.1007/s00060-020-7444-z.

Zaidi, S. S. et al. (2019): New plant breeding technologies for food security. In: Science 363(6434): 13901391. DOI: 10.1126/science.aav6316.

Zhang, Y. et al. (2020): A CRISPR way for accelerating improvement of food crops. In: Nature Food 1(4): 200-205. DOI: 10.1038/s43016-020-0051-8.

Zink, T./Geyer, R. (2017): Circular economy rebound. In: Journal of Industrial Ecology 21(3): 593-602. 